\title{
AUDITORIA CONCORRENTE NO CENTRO CIRÚRGICO: CONCEPÇÕES DOS ENFERMEIROS ${ }^{1}$
}

\section{Maíra Pereira de Souza}

Enfermeira; Especialista em Auditoria e Regulação em Saúde; Universidade do Extremo Sul de Santa Catarina (UNESC), Criciúma (SC), Brasil.

\section{Luciane Bisognin Ceretta}

Enfermeira; Doutora em Ciências da Saúde; Mestre em Enfermagem - Universidade do Extremo Sul de Santa Catarina (UNESC), Criciúma (SC), Brasil.

\section{Maria Tereza Soratto}

Enfermeira; Mestre em Educação; Docente no Curso de Enfermagem; Universidade do Extremo Sul de Santa Catarina (UNESC), Criciúma (SC), Brasil.

E-mail: guiga@unesc.net
RESUMO: A auditoria hospitalar desenvolveu-se com objetivo de responder às necessidades de calcular e analisar os custos da assistência prestada. Porém, atualmente, passou a ser compreendida como recurso gerencial, utilizado para qualificar a assistência. Pesquisa com objetivo de analisar as concepçóes dos enfermeiros sobre a auditoria concorrente realizada no Centro Cirúrgico. Pesquisa de abordagem qualitativa, descritiva, exploratória e de campo, a qual foi desenvolvida no Centro Cirúrgico e setor de Auditoria Interna de um hospital privado do sul de Santa Catarina através de entrevista semiestruturada com 05 enfermeiras auditoras. Concluiu-se que a auditoria concorrente no Centro Cirúrgico é uma ferramenta que visa auxiliar o processo de gestão, identificando e prevenindo possíveis falhas, bem como avaliando a qualidade da assistência prestada, proporcionando maior segurança e diminuição de gastos, o que beneficia tanto paciente quanto equipe profissional e instituição.

PALAVRAS-CHAVE: Administração Hospitalar; Auditoria de Enfermagem; Cirurgia.

\section{CONCURRENT AUDITORY IN THE OPERATION ROOM: NURSES' IDEAS ${ }^{1}$}

\begin{abstract}
Hospital auditory was developed to calculate and analyze the costs of the service. It is now considered an administrative resource to assess the quality of assistance. Current research analyzes nurses' concepts on concurring auditory in the Operation Room by a qualitative, descriptive, exploratory and field research, developed in the Operation Room and in the Internal Sector of a private hospital in the south of the state of Santa Catarina, Brazil, by a semi-structured interview with five auditory nurses. Concurrent auditory in the Operation Room is a tool for the benefit of the administration to identify and prevent possible faults and evaluate service quality for greater safety and cost decrease. It benefits the patient, the professional team and the institution.
\end{abstract}

KEY WORDS: Hospital administration; Nursing auditory; Surgery.

\section{INTRODUÇÃO}

A auditoria é considerada uma ferramenta importante para o desenvolvimento da qualidade e custos dos hospitais. A auditoria na

\footnotetext{
${ }^{1}$ Artigo baseado na Monografia de Pós-Graduação apresentada ao Curso de Especialização em Auditoria e Regulação em Saúde.
} 
Enfermagem tem como finalidade normatizar, orientar, disciplinar, racionalizar e identificar as deficiências existentes nos registros hospitalares, intervindo diretamente na conta do cliente evitando glosas desnecessárias principalmente nos setores mais críticos, como o Centro Cirúrgico (VIEIRA, 2014).

A auditoria tem sido um importante instrumento de apoio à gestão profissional das empresas de saúde, com os seus comentários e recomendações de melhoria, de redução de desperdícios e perdas, de fortalecimento dos controles internos e da diminuição ou eliminação dos riscos (VIEIRA, 2014).

A auditoria é uma atividade que avalia procedimentos, processos, sistemas, registros e documentos, com intuito de aferir o cumprimento dos planos, metas, objetivos e políticas da organização. São medidas que envolvem pessoas ligadas à execução de atividades operacionais nos diferentes setores do hospital, avaliando a sistemática vigente e a qualidade do serviço prestado (RODRIGUES, 2004; SILVA; ESPIRITO SANTO, 2013).

$\mathrm{Na}$ área da Enfermagem, a auditoria está em crescente expansão e a atuação do enfermeiro auditor tem como propósito otimizar os custos, evitar desperdícios e garantir que todos os procedimentos sejam realizados de forma adequada (SIQUEIRA, 2014). Diversos benefícios podem ser encontrados tanto para os pacientes, através da melhoria da qualidade na assistência, quanto para a equipe de Enfermagem, fornecendo dados para reflexão de sua própria prática profissional e também para a instituição, por permitir verificar o alcance de objetivos, dar continuidade a programação e auxiliar no controle de custos (KURCGANT, 2006).

Existe a necessidade de controle e acompanhamento contínuo dos processos de uma empresa, principalmente pelo acirramento da competitividade no mercado e pela globalização (FERREIRA; VENANCIO; ABRANTES, 2009). A necessidade crescente de ajustes e redução de custos em seus processos faz com que as instituições hospitalares passem a utilizar ferramentas que possibilitem a redução de custos, com a necessidade de controles internos mais apropriados, como a auditoria concorrente.

Um novo mercado se abre e muito vem sendo discutido, principalmente nas questões estratégicas de gerenciamento, controle de organizações de saúde e seus processos de gestão. Estas mudanças exigem do gerente a aquisição de novos conhecimentos e habilidades. Para que enfermeiros gerentes desenvolvam estas habilidades se faz necessário o conhecimento das técnicas e métodos gerenciais, em que a auditoria também faz parte do processo. Nas áreas administrativas os enfermeiros têm assumido muitos cargos nas diversas instituições de saúde e vêm ganhando espaços significativos.

Assim, este estudo justifica-se pela necessidade de explorar a auditoria concorrente e por acreditar que é uma atividade inerente ao processo de trabalho do enfermeiro e de suma importância, pois é o momento no qual são auditados os procedimentos durante e após terem acontecido. Neste caso, o auditor atua junto aos profissionais da assistência, a fim de monitorizar o estado clínico do paciente internado, verificando a procedência, as justificativas da internação, procedimentos, materiais, medicamentos, e qualidade da assistência prestada. É um trabalho realizado em tempo real, que colabora com o trabalho da auditoria interna da instituição, bem como de revisão de contas (GHIZONI; VUNHA; GIUSTINA, 2015).

A auditoria de Enfermagem concorrente no Centro Cirúrgico, como objeto do presente estudo, surge como uma opção para o controle eficiente das atividades, visando organização e melhoria contínua. Funcionando como ferramenta para o controle da organização, a auditoria concorrente, juntamente com a auditoria interna hospitalar, age como elemento primordial para o controle da organização como um todo. Nos dias atuais, a auditoria de Enfermagem no Centro Cirúrgico é utilizada pelas instituições de saúde com a finalidade de avaliar a assistência e otimizar os custos decorrentes da prestação dos serviços (OLIVEIRA; JACINTO; SIQUEIRA, 2013).

A auditoria de Enfermagem concorrente é uma área ainda pouco investigada, que serve como um instrumento de avaliação da qualidade da assistência nas instituições de serviços de saúde. O conhecimento sobre a auditoria de Enfermagem no Centro Cirúrgico e a compreensão do papel do enfermeiro é imprescindível para a qualidade da assistência (OLIVEIRA; JACINTO; SIQUEIRA, 2013; SIQUEIRA, 2014).

Nesta perspectiva este estudo teve por objetivo analisar as concepções dos enfermeiros sobre a auditoria concorrente realizada no Centro Cirúrgico. 


\section{MATERIAIS E MÉTODOS}

Pesquisa de abordagem qualitativa, descritiva, exploratória e de campo. $\mathrm{O}$ estudo foi desenvolvido no Centro Cirúrgico e setor de Auditoria Interna de um hospital privado do sul de Santa Catarina, sendo este uma instituição de médio porte exclusivamente privado, que atende somente convênios hospitalares.

Os dados foram coletados por meio de entrevista semiestruturada com 05 enfermeiros auditores atuantes na auditoria interna do hospital pesquisado. A entrevista foi realizada durante a jornada de trabalho com o levantamento do perfil das enfermeiras e as categorias norteadoras da pesquisa: concepção sobre auditoria concorrente; como é realizada a auditoria concorrente; os desafios e benefícios da auditoria concorrente; a avaliação da auditoria; as necessidades atuais para a melhoria e qualificação da auditoria concorrente no Centro Cirúrgico e sugestões sobre a temática.

A análise e interpretação dos dados foram realizadas pela análise de conteúdo, a partir da categorização dos dados, através da ordenação, classificação e análise final dos dados pesquisados (MINAYO, 2009).

Para preservar o sigilo e o anonimato dos sujeitos pesquisados, de acordo com as diretrizes e normas regulamentadoras da Resolução 466/12, utilizou-se indicador alfanumérico (E1 a E5). A pesquisa foi aprovada pelo Comitê de Ética em Pesquisa da UNESC pelo Parecer $\mathrm{n}^{\mathrm{o}} 1.027 .777 / 2015$.

\section{RESULTADOS E DISCUSSÃO}

\subsection{PERFIL DAS ENFERMEIRAS}

Todas as enfermeiras pesquisadas são do sexo feminino, idade variando entre 27 a 33 anos, com idade média de 30 anos; tempo de atuação na auditoria hospitalar de 1 ano e meio a 7 anos, com tempo médio de atuação de 4 anos e três meses. Quatro enfermeiras possuem especialização em Auditoria (E1, E2, E4 e E5). A Enfermeira E3 possui especialização em Obstetrícia e Neonatologia.

Os profissionais que atuam na auditoria em saúde devem possuir capacitação específica para a função
(SOUZA; DYNIEWICZ; KALINOWSKI, 2010). Em virtude das exigências do atual mercado na área da saúde, tornase primordial a qualificação do enfermeiro auditor para dar suporte à administração das instituições de saúde. A capacitação dos profissionais que atuam na auditoria hospitalar possibilita a melhoria e a eficiência do processo. $\mathrm{O}$ profissional deve se manter atualizado quanto às inovações na área de auditoria, construindo alicerce para uma atuação ética e de qualidade (SIQUEIRA, 2014).

O enfermeiro auditor é um profissional que deve ser um educador permanente da equipe de Enfermagem, buscando a qualificação do cuidado e do processo de auditoria (CARVALHO; SILVA, 2014).

\subsection{CONCEPÇÃO DOS ENFERMEIROS SOBRE AUDITO- RIA CONCORRENTE}

A auditoria concorrente tem por objetivo evitar o erro e a cobrança indevida das contas hospitalares. A auditoria hospitalar concorrente ocorre durante a internação até a alta do paciente, o que torna o processo mais ágil e eficaz (GHIZONI; VUNHA; GIUSTINA, 2015; SOUZA; DYNIEWICZ; KALINOWSKI, 2010). Esta concepção de auditoria foi destacada pelas enfermeiras auditoras da seguinte forma:

E1 - "Conferência do prontuário, alinbando o que o paciente recebe no atendimento ao que está sendo cobrado".

E2 - "Minha concepção sobre auditoria concorrente bospitalar tem por objetivo facilitar o diagnóstico e esclarecimento de registros durante a passagem do paciente no setor e, assim, conseguir ter mais precisão as cobranças na conta-paciente".

E3 - "Auditoria concorrente é a auditoria realizada dentro do setor em que o paciente está sendo atendido, onde o auditor tem contato direto com a equipe para esclarecer suas dúvidas".

E4 - "É a auditoria realizada de forma direta (in loco) no setor ao qual o paciente passou".

$\mathrm{E} 5$ - "É a auditoria que acontece muitas vezes antes da alta do cliente, podendo fazer a conferência dos materiais e medicamentos antes de fechar a conta". 
Percebe-se, conforme os relatos, que são distintas as concepções sobre auditoria para as enfermeiras pesquisadas. O conceito de auditoria foi proposto por Lambeck em 1956, tendo como premissa a avaliação de qualidade da atenção com base na observação direta, registros e história clínica do cliente, consistindo em uma forma de investigação com intuito de verificar o cumprimento de critérios e ações (SANTOS; SANTANA; VIEIRA, 2012).

A auditoria concorrente acontece durante o processo, para acompanhar a execução das atividades e garantir a qualidade do produto, sendo realizada quando o paciente ainda se encontra hospitalizado ou em atendimento ambulatorial (ANTONINI, 2011; MARQUIS; HUSTON, 2009; SOUZA; DYNIEWICZ; KALINOWSKI, 2010).

A auditoria concorrente envolve a avaliação feita pelo paciente e sua família, verificando suas percepções acerca da assistência prestada; entrevista e reflexão do profissional após a prestação do cuidado; exame do paciente e confronto com as necessidades levantadas; verificação do cumprimento das atividades a serem realizadas pelos profissionais e observação do ambiente (ANTONINI, 2011; SOUZA; DYNIEWICZ; KALINOWSKI, 2010).

Entende-se, então, que a auditoria é um exame minucioso, cuidadoso e sistemático das atividades desenvolvidas em uma empresa ou setor dela, com objetivo de verificar as disposições estabelecidas e se foram implementadas com eficácia e adequação.

Através da auditoria concorrente é possível, com maior exatidão, constatar irregularidades, tanto no prontuário médico, como também na área contábil, possibilitando a eliminação de desperdícios e falhas, simplificando tarefas, evitando retrabalho e transmitindo informações seguras sobre o desenvolvimento das atividades. Porém, para que este processo seja executado com excelência, considera-se imprescindível a adesão da equipe multiprofissional do Centro Cirúrgico para o alcance das metas da auditoria concorrente. Para tanto é necessário que a equipe conheça o que é auditoria concorrente, seus objetivos e propostas, além da otimização do processo de auditoria na instituição hospitalar. Somente com o trabalho de equipe, com informações e controle adequado do processo, é que será possível realizar a auditoria concorrente com qualidade e exatidão.

\subsection{REALIZAÇÃO DA AUDITORIA CONCORRENTE NO CENTRO CIRÚRGICO}

A auditoria hospitalar concorrente ocorre durante a passagem do paciente pelo setor do Centro Cirúrgico geralmente até a alta do paciente, o que facilita todo o processo de auditoria e faturamento da conta (SOUZA; DYNIEWICZ; KALINOWSKI, 2010).

E2 - "Fluxo=Contas da parte cirúrgica (todas). Contas do período matutino serão faturadas/auditadas à tarde, da tarde no próximo dia pela manbã. Noite e finais de semana deixar parte cirúrgica no setor de auditoria do CC. Contas ambulatoriais são fechadas 100\% e protocoladas diretamente para a auditoria".

$\mathrm{E} 3$ - "O auditor recebe a parte da conta hospitalar correspondente ao procedimento realizado e realiza a auditoria ainda com o atendimento do paciente em andamento ou se ambulatorial logo após o fechamento".

E4 - "Após a saída do paciente do setor, com suas devoluções realizadas, a auditora audita a conta e quando identifica divergências consegue fazer a atuação direta e rápida".

E5 - "A conta fica com a auditora do CC, ela confere e fecha a auditoria dela, e o prontuário é mandado para o faturamento para ser realizada a auditoria dos outros setores onde o paciente ficou".

Destaca-se na fala da enfermeira E1 a importância da educação contínua para a qualificação de todo o processo de auditoria concorrente no Centro Cirúrgico:

E1 - "Realizada auditoria imediata dos prontuários pós-procedimento cirúrgico, corrigindo falbas $e$ melhorando o processo com a educação diária dos colaboradores".

Realizar a auditoria concorrente é a melhor maneira para reduzir custos, evitar desperdícios e cobranças indevidas nas internações dos clientes que procuram o serviço, pois ela ocorre no momento em que o cliente ainda está no estabelecimento.

A auditoria concorrente ocorre no processo em que o paciente está em atendimento, envolvendo a aná- 
lise e avaliação dos registros de Enfermagem, entrevista com o cliente e/ou familiares, observação do cliente, exame físico e observação do ambiente (ANTONINI, 2011).

A auditoria concorrente em Centro Cirúrgico é muito mais que uma simples análise financeira. Ela é capaz de oferecer informações e subsidiar ações referentes à qualidade de assistência de Enfermagem:

a enfermeira auditora compete a garantia da qualidade da assistência prestada ao usuário, proporcionando-lhe confiabilidade e segurança na relação; viabilizar economicamente a empresa; efetuar levantamento dos custos assistenciais para determinar metas gerenciais e subsidiar decisões do corpo diretivo da empresa; fazer provisão e adequação dos materiais utilizados; conferir a correta utilização/cobrança dos recursos técnicos disponíveis; educar a operadora e os prestadores de serviços; proporcionar um espaço de diálogo permanente entre o prestador e a empresa e prestador/empresa/usuário (VIEIRA, 2014, p. 12).

A atribuição da enfermeira auditora é realizar avaliação do processo de assistência ao paciente e verificar a exatidão dos documentos relacionados a esta assistência (VIEIRA, 2014). Além disso, destaca-se a necessidade e importância da educação contínua, não apenas entre campos de saberes, mas entre os profissionais das diversas áreas da saúde, proporcionando a aquisição e troca de novos conhecimentos e desenvolvimento pessoal e profissional (SILVA; SEIFFERT, 2009).

\subsection{DESAFIOS PARA A IMPLANTAÇÃO DA AUDITORIA CONCORRENTE}

Dentre os desafios para a implantação da auditoria concorrente foram elencadas as mudanças de processos, a sistematização do fluxo entre faturamento, auditoria e mudança do fluxo, citadas pelas enfermeiras E3, E4 e E5. Destaca-se ainda a falta de experiência no relato da enfermeira E5.

E3 - "Mudanças de processos, instalações, poucas referências".
E4 - "Evidenciou que as dificuldades que tivemos no início foram a sistematização do fluxo entre faturamento e auditoria e os gastos realizados com a nova sala".

E5 - "Dificuldade foi a mudança de fluxo, contas que passaram e não foram entregues na auditoria do CC, que barramos no faturamento. Desafio foi a falta de experiência".

Houve resistência para a implantação da auditoria concorrente no Centro Cirúrgico por parte de alguns integrantes da equipe, segundo o relato das enfermeiras E1 e E2. Destaca-se na fala da enfermeira E2 como grande desafio o medo de não dar certo e não conseguir atingir as metas propostas, além da falta de profissionais no setor.

E1 - "Aceitação do serviço por parte dos colaboradores e médicos".

E2 - "O desafio maior foi o medo de não dar certo, da diminuição do quadro de colaboradores locado dentro do setor de auditoria e não conseguir atingir as metas colocadas pela diretoria. Dificuldades $=$ Resistên cia por parte de alguns integrantes dos setores afins com a mudança".

Na implantação de novos sistemas de trabalho pode ocorrer resistência dos profissionais com o novo processo. O enfermeiro auditor deve garantir a qualidade da assistência prestada ao usuário, sendo que um dos desafios para o desenvolvimento da auditoria em enfermagem é a descoberta de uma "identidade profissional" (VIEIRA, 2014, p. 12).

A adesão da equipe aos novos processos de trabalho, neste caso, a implantação da auditoria concorrente é um desafio necessário, pois o trabalho do enfermeiro auditor encontra-se em constante crescimento, na busca da qualificação da assistência.

O enfermeiro auditor precisa ser capaz de enfrentar os desafios da profissão, "buscando novas abordagens tais como a gerência participativa e os programas de qualidade, os quais são foco central do trabalho em auditoria" (VIEIRA, 2014, p. 11). 


\subsection{BENEFÍCIOS DA AUDITORIA CONCORRENTE NO CENTRO CIRÚRGICO}

A auditoria de Enfermagem é utilizada pelas instituições de saúde com a finalidade de avaliar a qualidade da assistência e acompanhar os custos decorrentes da prestação dos serviços, por meio da observação direta do registro e da história clínica do paciente (GHIZONI; VUNHA; GIUSTINA, 2015; OLIVEIRA; JACINTO; SIQUEIRA, 2013; VIEIRA, 2014).

A auditoria em saúde traz "benefícios para a instituição e para o paciente, pois visa uma maior qualidade da assistência prestada e satisfação do paciente/cliente, e despesa adequada e justa para instituição" (SIQUEIRA, 2014, p. 16).

Os benefícios da auditoria concorrente no Centro Cirúrgico envolvem a agilidade no processo, evitando o acúmulo de contas e retrabalho, diminuição de glosas e cobranças indevidas, com a descoberta de erros referentes a materiais e medicamentos no momento da própria auditoria, além da educação da equipe para o controle e continuidade do processo, conforme destacado nos relatos:

E1 - "Resolução rápida e precisa de possíveis erros no prontuário, agilizando o processo, evitando retrabalbo".

E2 - "Diminuição do retrabalbo, estar ativo no processo e precisão dos fatos $x$ clareza de registros (educação continuada)".

E3 - "Agilidade no processo, diminuição de glosas e retrabalbo, identificação de problemas durante o atendimento e educação constante entre profissionais".

E4 - "Resolve o problema no foco de forma ágil. Educa os colaboradores da área de saúde. Fica esclarecida e atualizada das novidades para a cobrança".

E5 - "Melhorou muito o faturamento das contas, pois se tem alguma coisa que não condiz com a conta, já é descoberto na hora e evitamos as cobranças indevidas e vice e versa".

De acordo com os comentários citados, percebese que muitos são os benefícios da auditoria concorrente no Centro Cirúrgico, sendo um dos principais o alerta para novos e antigos problemas ou deficiências, apontando alternativas de correções e/ou prevenções a partir de dados levantados com as análises realizadas durante a hospitalização do paciente, objetivando um resultado final com maior qualidade. Conforme Passos et al. (2012, p. 3), a auditoria concorrente ainda traz os seguintes benefícios:

[...] desenvolvimento dos indicadores da assistência, estabelecimento de critérios de avaliação e geração de novos conhecimentos; fiscalização de serviços realizados, qualidade desse serviço, análise sistemática do objeto da informação; cumprimento de normas institucionais e/ ou legais e avaliação comparativa entre assistência prestada, propiciando ainda ao auditor argumentar com os profissionais da assistência sobre o tratamento necessário ao paciente, o custo deste tratamento e, ainda, favorecer a definição de parâmetros e instrumentos adequados de avaliação.

O serviço de auditoria está associado ao controle administrativo-financeiro das instituições de saúde, erros em cobranças são relacionados à falha de registros de materiais e medicamentos utilizados e à ausência de anotações tanto pela equipe de Enfermagem quanto médica (OLIVEIRA; JACINTO; SIQUEIRA, 2013).

\footnotetext{
Especificamente, a auditoria no Centro Cirúrgico reflete diretamente o serviço de enfermagem, pois, a partir das anotações e cuidados prescritos, são justificados os equipamentos e gases (oxigênio, ar comprimido, halotano, etc.) utilizados com o débito assinalado na nota de gasto do Centro Cirúrgico (OLIVEIRA; JACINTO; SIQUEIRA, 2013, p. $155)$.
}

"A auditoria de Enfermagem, como processo educativo fornece importantes subsídios para a implantação e gerenciamento de uma assistência de enfermagem mais segura, ágil, criativa, eficaz e eficiente" (VIEIRA, 2014, p. 11). 


\subsection{AVALIAÇÃO DA AUDITORIA CONCORRENTE NO CENTRO CIRÚRGICO APÓS O PROCESSO DE IM- PLANTAÇÃO}

As enfermeiras fazem uma boa avaliação do processo de implantação da auditoria concorrente no Centro Cirúrgico, com maior agilidade em todo o processo - faturamento $\mathrm{x}$ auditoria, trazendo maior autonomia e entrosamento entre a equipe multiprofissional.

E1 - "Ainda está no processo de implantação e as mudanças e melhorias ocorrem diariamente. Mas o serviço vem nos mostrando eficiência e hoje é considerado indispensável, devido à agilidade nas resoluções de falhas no processo".

E2 - "Agilidade no processo, trabalha os problemas de imediato (resolutividade e educação) e auditorias mais autônomas e atuantes".

E3 - "Agilidade no processo, diminuição de retrabalbo e entrosamento entre setores".

E4 - "Favoreceu muito nosso trabalbo, pois não ficamos com a conta parada, temos mais acesso aos profissionais de saúde para educar. Mantemos as contas em dia".

E5 - "Melbora significante nas contas que ficavam paradas aguardando ajustes, boje isto não acontece. E quando acontece temos alguém lá no CC para estar fazendo esta ponte. As contas não ficam paradas".

Foi possível identificar avaliações positivas pelas enfermeiras após o processo de implantação da auditoria concorrente no Centro Cirúrgico, pois proporcionou maior agilidade no trabalho e na resolução das falhas, autonomia e melhor entrosamento entre setores. Conforme Menezes (2011, p. 70), a implantação da auditoria concorrente é de grande valia, pois

[...] possibilita o desenvolvimento de indicadores de assistência, estabelecendo critérios de avaliação e consequentemente geração de novos conhecimentos, o que possibilita uma avaliação dos problemas enfrentados e as diversas condutas adotadas para cada um deles, e a solução encontrada para cada um dos problemas. Além disso, analisa a qualidade da assistência ofertada, como também os gastos efetuados na realização dos atendimentos e o controle, que muito contribui para promover, tanto a redução dos custos, quanto a melhoria dos processos, tendo como maior foco o cidadão que precisa de atendimento.

$\mathrm{Na}$ gestão hospitalar, a auditoria se destaca como instrumento de fiscalização e controle mais adequado às necessidades de gerenciamento das informações subsidiando a tomada de decisões (VIEIRA, 2014). A avaliação da auditoria concorrente no Centro Cirúrgico é realizada através de indicadores mensais, com reajuste de contas com problemas e de treinamentos individuais com a equipe multidisciplinar.

\subsection{AS NECESSIDADES ATUAIS PARA A MELHORIA E QUA- LIFICAÇÃO DA AUDITORIA CONCORRENTE NO CENTRO CIRÚRGICO}

A necessidade atual para a melhoria e qualificação da auditoria concorrente no Centro Cirúrgico depende da aceitação e empenho de toda a equipe multiprofissional no novo processo que envolve a auditoria concorrente:

E1 - "Entendimento e aceitação de todos os envolvidos que a auditoria concorrente veio para acrescentar e não para fazer cobranças apenas. O objetivo é que todos cresçam com o trabalbo em conjunto".

E2 - "Descobrir todas as falbas do processo para trabalbar cada caso na sua base".

E3 - "Conscientização na parceria entre auditor e equipes".

E4 - "Os profissionais de saúde saberem a importância do profissional auditor para um equilíbrio financeiro e assistencial".

E5 - "Ainda é o fluxo por que alguns funcionários ainda não entenderam o processo, e acabam esquecendo de deixar a parte do CC com a auditoria, e isso acaba atrapalbando o processo".

Pode-se considerar a auditoria como um processo de avaliação para o redirecionamento das ações, sendo que após a análise do serviço e a verificação das 
deficiências o enfermeiro auditor pode tomar decisões corretivas e/ou preventivas para remodelar essas ações (CARVALHO; SILVA, 2014). A enfermeira no processo de auditora tem possibilidade de qualificar o gerenciamento dos recursos e atingir metas previamente estabelecidas (VIEIRA, 2014).

\subsection{SUGESTÕES SOBRE A TEMÁTICA}

As enfermeiras consideram imprescindível a ampliação do processo de auditoria concorrente nos demais setores do hospital:

E2 - "Ampliar nos demais setores do bospital".

E4 - "Que seja implantado aos outros setores".

E5 - "Implantar auditoria concorrente em todos os setores do bospital, seria muito bom".

A enfermeira E3 considera importante a realização de uma nova pesquisa após a implantação de todo o processo:

E3 - "Aplicar nova pesquisa após um tempo maior de implantação para comparar resultados".

A Auditoria Concorrente é considerada uma nova forma de auditar, sendo necessário o trabalho em equipe para evitar falhas durante o processo e a agilidade no fechamento das contas:

E1 - "Acredito que estamos no caminbo certo, moldando um serviço com o perfil da instituição. Uma nova forma de auditar está surgindo e estamos nos adaptando. Agora devemos trabalhar intensamente com todos os envolvidos no processo cirúrgico e alinharmos um novo jeito de trabalhar em equipe para evitar a falha".

A auditoria é considerada elemento essencial para a mensuração da qualidade da assistência à saúde oferecendo subsídios à equipe multiprofissional para reorientar suas atividades, estimulando a reflexão individual e coletiva e nortear o processo de educação permanente (CARVALHO; SILVA, 2014).
$\mathrm{Na}$ área da Enfermagem, a auditoria está em crescente expansão, sendo uma tendência de mercado, tendo variadas abordagens: auditor de contas, qualidade da assistência na pesquisa e processos. A atuação do enfermeiro auditor tem grande importância quando o propósito é otimizar os custos, evitar desperdícios, garantir que todos os procedimentos sejam realizados de forma adequada (SIQUEIRA, 2014; CARVALHO; SILVA, 2014; VIEIRA, 2014). Visualizam-se novas perspectivas para a atuação do enfermeiro auditor tendo como embasamento um saber e um fazer crítico reflexivo, fundamental para a qualificação técnica e ética do cuidado (VIEIRA, 2014, p. 11).

\section{CONSIDERAÇÕES FINAIS}

$\mathrm{O}$ resultado da pesquisa denotou os benefícios que a auditoria de Enfermagem concorrente realizada no Centro Cirúrgico traz para a auditoria interna hospitalar, em relação ao faturamento da conta após a alta do cliente, sendo mais difícil realizar a auditoria no prontuário. Desta forma a auditoria concorrente ganha seu espaço, sendo que a conta já sai faturada e auditada do Centro Cirúrgico, evitando retrabalho para o restante da equipe de enfermeiros auditores, que irão dar continuidade ao processo de auditoria até o arquivamento do prontuário.

A auditoria concorrente no Centro Cirúrgico ocorre quando o paciente ainda está internado na unidade, envolvendo a análise e avaliação dos registros de Enfermagem, entrevista com o paciente e/ou familiares, observação do paciente, exame físico e observação do ambiente, o que possibilita o desenvolvimento de indicadores de assistência, estabelecendo critérios de avaliação e, consequentemente, a geração de novos conhecimentos, favorecendo a avaliação das possíveis falhas. Sugere-se um aprofundamento da pesquisa sobre o gerenciamento de Enfermagem e a auditoria.

\section{REFERÊNCIAS}

ANTONINI, C. N. Avaliação do processo de um serviço de auditoria interna em um hospital de grande porte. 2011. 29 f. Trabalho de Conclusão de Curso (Auditoria e 
Gestão de Saúde). Curitiba, PR. Disponível em: < http:// tcconline.utp.br/wp-content/uploads/2011/10/AVALIACAO-DO-PROCESSO-DE-UM-SERVICO-DE-AUDITORIA -INTERNA-EM-UM-HOSPITAL-DE-GRANDE-PORTE.pdf $>$. Acesso em: 12 jan. 2016.

BRASIL. Conselho Nacional de Saúde. Resolução no 466, de 12 de dezembro de 2012. Disponível em: < http:// conselho.saude.gov.br/resolucoes/2012/Reso466.pdf > . Acesso em: 10 mar. 2015.

CARVALHO, F. A.; SILVA, M. A. A Auditoria de enfermagem como possibilidade da melhoria do Cuidado. Estudos, Goiânia, v. 41, especial, p. 43-58, out. 2014.

FERREIRA, M. A. M.; VENANCIO, M. M.; ABRANTES, L. A. Análise da eficiência do setor de supermercados no Brasil. Econ. Apl., Ribeirão Preto, v. 13, n. 2, p. 333-347, jun. 2009. Disponível em: <http:// www.scielo.br/scielo.php? script $=$ sci_arttext $\&$ pi$\mathrm{d}=$ S1413-80502009000200007\&lng $=$ en\&nrm $=$ iso $>$. Acesso em: 06 jul. 2016. http://dx.doi.org/10.1590/ S1413-80502009000200007.

GHIZONI, M. W.; VUNHA, K. P.; GIUSTINA, K. P. D. Atuação do enfermeiro que pratica auditoria hospitalar em um hospital de grande porte da região sul de Santa Catarina. Rev. Ciênc. Cidadania, v. 1, n. 1, 2015.

KURCGANT, P. Administração em enfermagem. São Paulo: EPU, 2006.

MARQUIS, B. L.; HUSTON, C. J. Administração e liderança em enfermagem: teoria e prática. 6 . ed. Porto Alegre: Artmed, 2009.

MENEZES, J. G. B. R. L. Auditoria em enfermagem: um instrumento para assistência de qualidade. Rev Enferm. UNISA, v. 12, n. 1, 2011. Disponível em: < http:// www.unisa.br/graduacao/biologicas/enfer/revista/ arquivos/2011-1-12.pdf > . Acesso em: 12 jan. 2016.

MINAYO, M. Pesquisa social: teoria, método e criatividade. Rio de Janeiro: Vozes, 2009.

OLIVEIRA, D. R.; JACINTO, S. M.; SIQUEIRA, C. L. Auditoria de enfermagem em Centro Cirúrgico. RAS, v. 15, n. 61, out./dez. 2013.
PASSOS, M. L. L. et al. Auditoria de enfermagem: conhecimento de profissionais em hospital público de referência. Rev Rene, v. 13, n. 5, 2012. Disponível em: <http://www.revistarene.ufc.br/revista/index.php/ revista/article/view/1160/pdf > . Acesso em: 17 mar. 2015.

RODRIGUES, V. A. Glosas hospitalares: importância das anotações de enfermagem. Rev Arq Ciência da Saúde, v. 11,n. 4, 2004. Disponívelem: < http://www.rdconsultoria. com.br/Downloads/Educa\%C3\%A7\% $\quad$ C3\%A30\%20 Continuada/Administra\%C3\%A7\%C3\%A30\% 20 Hospitalar/Glosas\%20Hospitalares.pdf $>$. Acesso em: 18 mar. 2015.

SANTOS, C. A.; SANTANA, É. J. S.; VIEIRA, R. P. A auditoria e o enfermeiro como ferramentas de aperfeiçoamento do SUS. Rev Baiana de Saúde Pública, v. 36, n. 2, p. 539559, abr./jun. 2012. Disponível em: < http://inseer.ibict. br/rbsp/index.php/rbsp/article/viewFile/475/pdf_151>. Acesso em: 12 jan. 2016.

SILVA, A. T.; ESPIRITO SANTO, E. A auditoria como ferramenta para a excelência da gestão hospitalar. Rev Saúde e Desenvolvimento, v. 3, n. 2, jan.jun. 2013. Disponível em: < http://www.grupouninter.com.br/revistasaude/ index.php/saudeDesenvolvimento/article/view/57/109>. Acesso em: 12 jan. 2016.

SILVA, G. M.; SEIFFERT, O. M. L. B. Educação continuada em enfermagem: uma proposta metodológica. Rev Bras Enferm., n. 62, v. 3, Brasília, maio/jun., 2009. Disponível em: <http://www.scielo.br/pdf/reben/v62n3/05.pdf > . Acesso em: 12 jan. 2016.

SIQUEIRA, P. L. F. Auditoria em Saúde e atribuições do Enfermeiro Auditor. Cad Saúde e Desenvolvimento, v. 3, n. 2, jul./dez. 2014.

SOUZA, L. A. A.; DYNIEWICZ, A. M.; KALINOWSKI, L. C. Auditoria: uma abordagem histórica e atual. Rev Adm Saúde., v. 12, n. 47, p. 71-8, 2010.

VIEIRA, A. P. T. Enfermeira auditora uma ferramenta importante para a qualidade do serviço em saúde em hospitais privados. Rev Especialize On-line IPOG, Goiânia, v. 1, n. 9 , p. 1-13, dez./2014. 
Recebido em: 02 de fevereiro de 2016

Versão final recebida em: 07 de julbo de 2016

Aceito em: 21 de julbo de 2016 